\title{
HIV persistence: clonal expansion of cells in the latent reservoir
}

\author{
Kyungyoon J. Kwon' and Robert F. Siliciano ${ }^{1,2}$ \\ 'Department of Medicine, Johns Hopkins University School of Medicine, Baltimore, Maryland, USA. ${ }^{2}$ Howard Hughes Medical Institute, Baltimore, Maryland, USA.
}

\begin{abstract}
While antiretroviral therapy (ART) can reduce HIV-1 to undetectable levels, the virus generally reappears if treatment is stopped. Resurgence of the virus is due to the reactivation of $\mathrm{T}$ cells harboring latent integrated provirus, and recent studies indicate that proliferation of these latently infected cells helps maintain the HIV-1 reservoir. In this issue of the $J C I$, Lee et al. evaluated $\mathrm{CD4}^{+} \mathrm{T}$ cell subsets to determine whether certain populations are more likely to harbor full-length, replication-competent provirus. The authors identified an enrichment of clonally expanded Th1 cells containing intact HIV-1 proviruses, suggesting that this polarized subset contributes to the persistence of the reservoir. Strategies to target these provirus-harboring cells need to be considered for future therapies aimed toward HIV-1 cure.
\end{abstract}

\section{Targeting the latent HIV-1} reservoir: challenge for a cure Despite the efficacy of antiretroviral therapy (ART) in suppressing HIV-1 replication, there is still no cure for HIV-1 infection due to the presence of a latent reservoir for the virus (1). This latent reservoir consists mainly of resting memory $\mathrm{CD} 4^{+}$ $\mathrm{T}$ cells harboring integrated HIV-1 proviruses. The reservoir has an extremely slow decay rate such that viral eradication by ART alone is not possible (1). Recent studies have shown that one of the major mechanisms of reservoir persistence is the clonal expansion of these latently infected cells. Initial evidence for this route of persistence came from the independent detection of multiple proviruses with exactly the same viral sequence or integration site within host cells (2-6). Full genome sequencing studies have established that most proviruses present in resting $\mathrm{CD} 4^{+} \mathrm{T}$ cells are defective (6-8), and thus many of these proviruses are unlikely part of the latent reservoir. Nevertheless, recent work from several groups has demonstrated that cells carrying replication-competent proviruses can also clonally expand in vivo (9-12). The proliferation of cells carrying intact, replication-competent proviruses is a troubling finding that helps explain the stability of the HIV-1 reservoir and raises concerns about the feasibility of eradication.

\section{Growing evidence for clonal expansion}

Clonal expansion is a basic aspect of normal $\mathrm{T}$ cell biology. Antigen-driven proliferation and cytokine-driven homeostatic proliferation are well established as mechanisms by which $\mathrm{T}$ cell populations expand (13). However, it has been less clear whether or not infected $\mathrm{CD}^{+} \mathrm{T}$ cells can proliferate, especially as productively infected cells have a very short in vivo half-life (14, 15). Early evidence for clonal expansion of HIV-1-infected cells came from studies of residual viremia, the trace level of free virus detectable in the plasma of treated patients with special methods. Despite the extensive viral sequence diversification that occurs over time in untreated patients, the residual viremia observed once patients start a suppressive ART regimen is often surprisingly oligoclonal, suggesting that it is produced by expanded cellu-

Related Article: p. 2689

Conflict of interest: The authors have declared that no conflict of interest exists

Reference information: J Clin Invest. 2017;127(7):2536-2538. https://doi.org/10.1172/JCI95329.

lar clones carrying exactly the same proviruses $(16,17)$. However, an alternative explanation for viral sequence identity is that multiple cells are infected by a single dominant viral variant. Definitive proof that clonal expansion of infected cells has occurred can be obtained by demonstrating that these cells carry the same proviral sequence integrated at the same exact position in the human genome. After the development of next-generation sequencing technologies that allowed for efficient integration site sequencing, several groups reported the detection of expanded clones based on the presence of identical integration sites (3-5); however, integration site analysis captures only the very end of the viral genome. Given that the majority of proviruses are defective, these studies establish the clonal expansion of infected cells but do not necessarily identify those carrying intact viral genomes. One interesting concept to come out of these studies was the idea that proviral integration into particular host genes might alter expression of those genes in a way that promotes cell proliferation and/or survival $(2,3)$.

Recently, several groups have provided evidence for the clonal expansion of cells carrying replication-competent proviruses (9-12). Simonetti and colleagues identified an integration site associated with a single, dominant $\mathrm{CD}^{+}{ }^{+} \mathrm{T}$ cell clone carrying a replication-competent provirus in an HIV1-infected patient with squamous cell carcinoma (9). Interestingly, the clonally expanded $\mathrm{T}$ cells were found at sites of the disseminated malignancy in this patient, raising the possibility of antigendriven expansion. In addition, three groups recently showed that multiple $\mathrm{CD}^{+}$ $\mathrm{T}$ cells carrying identical, replicationcompetent proviruses are often present in a single blood sample from treated patients (10-12). In all three studies, the fraction of viral isolates that had sequence exactly matching another independent isolate from the same sample was over $50 \%$. With additional sampling, it is likely that 
A

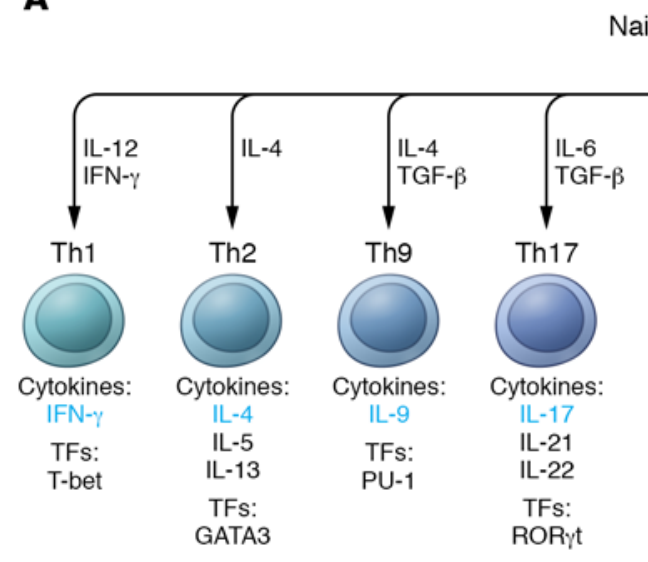

B

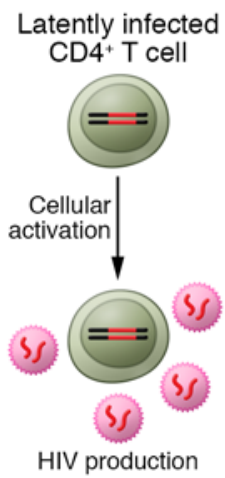

C

Replication-competent provirus in differentiated $\mathrm{T}$ cell subset

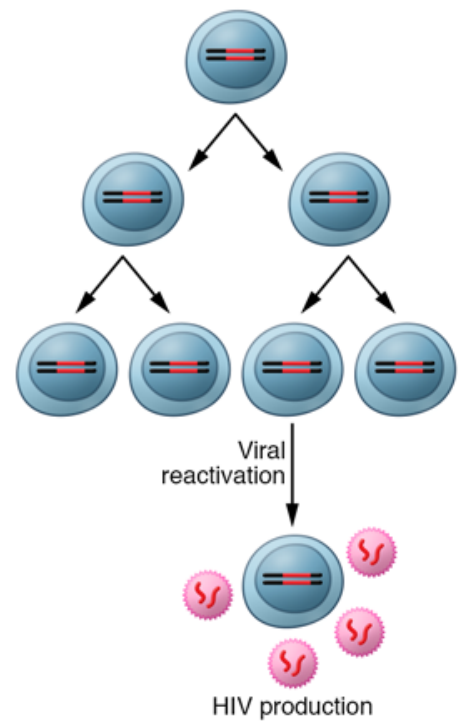

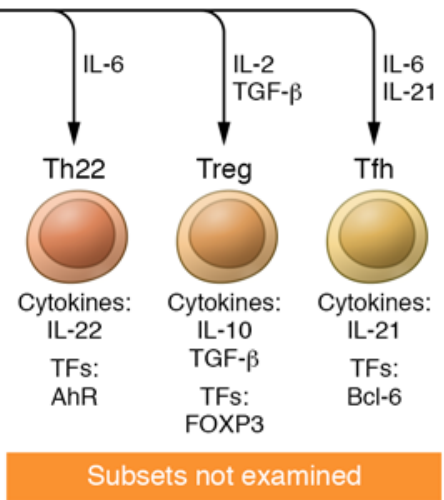

D

Defective provirus in differentiated T cell subset

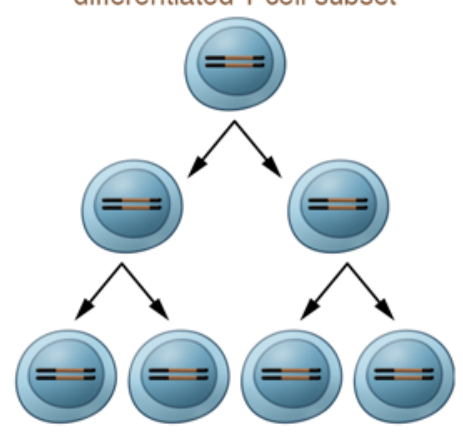

Figure 1. Intact, clonally expanded HIV-1 proviruses in Th1 subpopulation. (A) $C D 4^{+} T$ cells differentiate into functional subsets, each of which is characterized by a defining transcription factor (TF) and a set of cytokines produced following activation. In this issue, Lee et al. sorted peripheral blood mononuclear cells from 3 cART-treated patients into $\mathrm{CD}^{+}{ }^{+} \mathrm{T}$ cell subpopulations based on production of 4 signature cytokines (blue). Subsets not examined are labeled as such. Cellular activation induces virus production from latently infected $\mathrm{CD}^{+}$ T cells (B). Differentiated T cell subsets carrying replication-competent (C) or defective (D) proviruses can also be stimulated to proliferate. In the patients studied by Lee et al., clonally expanded, intact proviral sequences were predominantly in the largest subset, Th1 cells. See Supplemental Table 3 in ref. 23. Tfh, $T$ follicular helper. matching sequences could be found for most if not all of these isolates. Together, these findings suggest that the majority of cells constituting the latent reservoir are generated by the proliferation of a smaller number of previously infected cells rather than by direct infection. These results do not support the idea that de novo infection events are a major factor in reservoir stability, as has been recently suggested (18). Rather it appears that the remarkable stability of the latent reservoir is largely due to cellular proliferation.

How can we reconcile the strong evidence for proliferation with the short in vivo half-life of productively infected cells? In the studies described above, the clonal $\mathrm{T}$ cell populations carrying replication-competent proviruses were demonstrated using variations of the viral outgrowth assay in which limiting dilutions of resting $\mathrm{CD} 4^{+} \mathrm{T}$ cells are activated with a mitogen, and replicationcompetent viruses are allowed to grow out. In one study, restimulation of cultures that were negative for viral outgrowth, despite uniform $\mathrm{T}$ cell activation with mitogen, resulted in additional outgrowth of replication-competent viruses (12). These findings show that $\mathrm{CD} 4^{+} \mathrm{T}$ cells carrying replication-competent proviruses can proliferate without producing virus while retaining the ability to do so upon subsequent stimulation (12). Thus, proliferation need not be limited by the short half-life of productively infected cells. It is unclear what prevents some latent proviruses from being induced upon cellular activation. The fact that cells carrying replication-competent proviruses can multiply while evading immune recognition presents a major challenge to current cure strategies and attempts to eradicate the latent reservoir.

\section{The role of proliferation in persistence of the reservoir}

The normal decay of cells in the latent reservoir may be balanced by proliferation of cells with latent provirus, thus resulting in the long observed half-life of the reservoir $(19,20)$. However, the dynamics of various subpopulations of $\mathrm{CD}^{+} \mathrm{T}$ cells in the context of HIV-1 infection are not well understood, and most studies are limited to peripheral blood samples. More studies 
on the proliferation of various populations of infected cells in tissue compartments would be beneficial in determining the natural dynamics of the latent reservoir. Latent proviruses are present in various $\mathrm{CD}^{+} \mathrm{T}$ cells subsets $(13,21,22)$. For example, previous studies from Lichterfeld and colleagues have described the presence of proviral DNA in a small, long-lived population of CD4 ${ }^{+}$memory $\mathrm{T}$ cells with stem cell properties (21). In this issue of the JCI, Lee et al. apply a full genome sequencing approach to evaluate functional subsets of $\mathrm{CD}^{+} \mathrm{T}$ cells (23). Cells were sorted based on the production of signature, subset-defining cytokines, and cellular DNA was subjected to full genome sequencing of the proviruses present. Consistent with previous studies, Lee et al. found that the vast majority of proviruses are defective. They also determined that independent sequences that were identical to other sequences from the same patient constitute over half of all intact sequences detected, consistent with previous studies of replication-competent isolates (23). Importantly, Lee et al. report that there is an enrichment of clonally expanded intact proviruses in functionally polarized Th1 cells (23), as depicted in Figure 1, adding to Lichterfeld and colleagues' previous findings of enriched integration in Th1 cells (22). While clonal expansion of cells harboring defective proviruses was also observed, intact proviruses were enriched in circulating Th1-polarized cells. Lee and colleagues propose that the virus may exploit the relatively high proliferation rate of Th1 cells to propagate via the division of infected host cells (23). It would be of interest to compare the proliferation and dynamics of infected and uninfected Th1 cells to further assess the role of proliferation of specific functional subsets in viral persistence. If the reservoir is largely maintained by natural host physiological mechanisms, it may be difficult to develop cure strategies that block proliferation without affecting normal $\mathrm{T}$ cell homeostasis.

\section{Concluding remarks}

Overall, the study by Lee et al. adds to the growing consensus that persistence of the latent reservoir of HIV-1 may be due in large part to the clonal expansion of cells harboring intact, replication-competent virus. Both homeostatic, cytokine-driven proliferation and antigen-stimulated prolif- eration may contribute to viral persistence, and both forms of proliferation have been shown to occur without reactivation of latent proviruses $(12,24)$. In either case, it may be difficult to block proliferation and reduce the size of the latent reservoir without creating negative consequences for patients. Here, methods to selectively block proliferation may be key. A growing number of studies and several clinical trials are evaluating immunosuppressive agents that may block proliferation and decrease the number of cells carrying potentially infectious proviruses. Recent clinical studies involve immunosuppressants such as tacrolimus or sirolimus that are usually administered in the setting of organ transplantation. There is evidence for decreased levels of HIV-1 DNA in patients treated with some immunosuppressants (25). Further investigation of the direct effects of immunosuppressants on the reservoir are needed to determine whether blocking proliferation might be a possible adjunct strategy in the search for a cure.

Address correspondence to: Robert F. Siliciano, Room 879, Edward D. Miller Research Building, 733 N. Broadway, Baltimore, Maryland 21205, USA. Phone: 410.955.2958; Email: rsiliciano@jhmi.edu.

1. Finzi D, et al. Latent infection of $\mathrm{CD} 4^{+} \mathrm{T}$ cells provides a mechanism for lifelong persistence of HIV-1, even in patients on effective combination therapy. Nat Med.1999;5(5):512-517.

2. Maldarelli F, et al. HIV latency. Specific HIV integration sites are linked to clonal expansion and persistence of infected cells. Science. 2014;345(6193):179-183.

3. Wagner TA, et al. HIV latency. Proliferation of cells with HIV integrated into cancer genes contributes to persistent infection. Science. 2014;345(6196):570-573.

4. Cohn LB, et al. HIV-1 integration landscape during latent and active infection. Cell. 2015;160(3):420-432.

5. von Stockenstrom S, et al. Longitudinal genetic characterization reveals that cell proliferation maintains a persistent HIV type 1 DNA pool during effective HIV therapy. JInfect Dis. 2015;212(4):596-607.

6 . Bruner KM, et al. Defective proviruses rapidly accumulate during acute HIV-1 infection. Nat Med. 2016;22(9):1043-1049.

7. Ho YC, et al. Replication-competent noninduced proviruses in the latent reservoir increase barrier to HIV-1 cure. Cell. 2013;155(3):540-551.

8. Imamichi H, et al. Defective HIV-1 proviruses produce novel protein-coding RNA species in HIV-infected patients on combination antiretroviral therapy. Proc Natl Acad Sci US A. 2016;113(31):8783-8788.
9. Simonetti FR, et al. Clonally expanded CD $4^{+} \mathrm{T}$ cells can produce infectious HIV-1 in vivo. Proc Natl Acad Sci U S A . 2016;113(7):1883-1888.

10. Lorenzi JC, et al. Paired quantitative and qualitative assessment of the replication-competent HIV-1 reservoir and comparison with integrated proviral DNA. Proc Natl Acad Sci US A. 2016;113(49):E7908-E7916.

11. Bui JK, et al. Proviruses with identical sequences comprise a large fraction of the replication-competent HIV reservoir. PLoS Pathog. 2017;13(3):e1006283.

12. Hosmane NN, et al. Proliferation of latently infected CD4(+) T cells carrying replicationcompetent HIV-1: Potential role in latent reservoir dynamics. JExp Med. 2017;214(4):959-972.

13. Chomont N, DaFonseca S, Vandergeeten C, Ancuta P, Sékaly RP. Maintenance of $\mathrm{CD} 4^{+}$ T-cell memory and HIV persistence: keeping memory, keeping HIV. Curr Opin HIV AIDS. 2011;6(1):30-36.

14. Wei X, et al. Viral dynamics in human immunodeficiency virus type 1 infection. Nature. 1995;373(6510):117-122.

15. Perelson AS, et al. Decay characteristics of HIV-1-infected compartments during combination therapy. Nature. 1997;387(6629):188-191.

16. Bailey JR, et al. Residual human immunodeficiency virus type 1 viremia in some patients on antiretroviral therapy is dominated by a small number of invariant clones rarely found in circulating $\mathrm{CD}^{+} \mathrm{T}$ cells. J Virol. 2006;80(13):6441-6457.

17. Tobin NH, et al. Evidence that low-level viremias during effective highly active antiretroviral therapy result from two processes: expression of archival virus and replication of virus. $J$ Virol. 2005;79(15):9625-9634.

18. Lorenzo-Redondo R, et al. Persistent HIV-1 replication maintains the tissue reservoir during therapy. Nature. 2016;530(7588):51-56.

19. Farber DL, Yudanin NA, Restifo NP. Human memory T cells: generation, compartmentalization and homeostasis. Nat Rev Immunol. 2014;14(1):24-35.

20. Murray AJ, Kwon KJ, Farber DL, Siliciano RF. The latent reservoir for HIV-1: how immunologic memory and clonal expansion contribute to HIV-1 persistence. J Immunol. 2016;197(2):407-417.

21. Buzon MJ, et al. HIV-1 persistence in $\mathrm{CD}^{+} \mathrm{T}$ cells with stem cell-like properties. Nat Med. 2014;20(2):139-142.

22. Sun $\mathrm{H}$, et al. Th1/17 polarization of CD4 T cells supports HIV-1 persistence during antiretroviral therapy. J Virol. 2015;89(22):11284-11293.

23. Lee GQ, et al. Clonal expansion of genome-intact HIV-1 in functionally polarized Th1 CD $4^{+} \mathrm{T}$ cells. JClin Invest. 2017;127(7):2689-2696.

24. Bosque A, Famiglietti M, Weyrich AS, Goulston C, Planelles V. Homeostatic proliferation fails to efficiently reactivate HIV-1 latently infected central memory CD4 ${ }^{+}$T cells. PLoS Pathog. 2011;7(10):e1002288.

25. Delagrèverie HM, Delaugerre C, Lewin SR, Deeks SG, Li JZ. Ongoing clinical trials of human immunodeficiency virus latency-reversing and immunomodulatory agents. Open Forum Infect Dis. 2016;3(4):ofw189. 